\title{
A Cooperative Game Framework for the Joint Operation of Natural Gas Storage and Electric Power Generation
}

\author{
Yucheng $\mathrm{Wu}$ \\ Pennsylvania State University \\ yzw124@psu.edu
}

\begin{abstract}
We develop a static cooperative game-theoretic framework for analyzing the impact of natural gas storage on interconnected gas and electricity markets. While increased utilization of gas storage has been proposed as a policy solution to fuel-security concerns in the electric power grid, the mode of interaction between gas storage units and electric power markets has not been investigated and some potential for cross-market manipulation exists. We investigate the potential for collusive behavior between gas storage units and power plants, whereby joint profits in the electricity and gas markets are increased by a strategy that involves the cooperative agents taking a loss in one market to the benefit of the other market. A cooperative strategy increases joint profits in scenarios when peak demand natural gas prices are high and the power plant(s) involved in the cooperative arrangement have relatively low marginal costs. The value of cooperation is not affected by whether gas storage units are physically connected to gas-fired power plants or if gas storage units inject gas into existing pipeline systems. While additional research into the nature of these competitive effects is needed, particularly in a repeated game context, our results point to the need to carefully consider the competitive effects of fuel security measures. A mechanism for monitoring of interactions between gas storage and power plants is likely warranted.
\end{abstract}

\section{Introduction}

For economic and environmental reasons, the utilization of natural gas in the electric power has been increasing over the past decade in the United States and elsewhere. While this fuel transition has brought benefits in the form of lower emissions of greenhouse gases and other pollutants, it has also brought a tighter coupling between electricity and natural gas markets. Since more than $30 \%$ of power generation in the

\author{
Seth Blumsack \\ Pennsylvania State University and Santa Fe Institute \\ sab51@psu.edu
}

Northeast U.S. is gas-fired, natural gas transmission in this region is constrained when both residential and power sector are at peak demand. The resulting impacts on natural gas market prices and threats to the reliability of the bulk power system have raised the profile of fuel security as an important policy issue affecting both the gas and electric transmission systems.

Recent work $[1,2,3,4]$ has focused on joint evaluations of electricity and natural gas markets for planning and market coordination purposes. One specific mechanism proposed for addressing fuel security concerns is the use of on-site fuel storage at natural gas power plants $[1,5,6]$. Such fuel storage appears to be an economical way to mitigate some fuel security concerns, but also tightens the coupling between natural gas and electricity markets.

The present paper focuses on the potential competitive effects of this inter-market coupling, particularly the potential for natural gas storage to be a strategic player in electricity markets. Our game-theoretic approach is in line with previous studies of vertical integration and strategic behavior issues that have been conducted within the natural gas sector [7, 8, 9] as well as related to electric power systems $[10,11,12,13]$. A major difference with this analysis, however, is in the application to cross-market integration and the use of a cooperative game-theoretic framework $[13,14]$. We build on prior work $[15,16,17]$ to model possible cooperation strategies between natural gas storage asset and power generation firms. These coalitions can impact the behavior of natural gas suppliers, transmission system operators, power generators, and electricity market clearing.

In this paper, section 2 introduces a simple static model of electricity and natural gas markets with power generation technologies, gas storage operators and power grid operators as types of players in the game. Two markets are connected at this single node with three power plants and one natural gas supplier. Section 3 gives two examples. One is a simple numerical 
example helping understand the model. Another one is en empirical example using empirical gas market data. These examples illustrate more general conditions under which we would expect coalition-forming between gas storage and electric power supply to be jointly profitable. Section 4 offers some thoughts on future research directions and policy lessons.

\section{Model Structure and Cooperative Cases}

Our modeling framework features an interconnected natural gas and electricity market. The natural gas market clears in a decentralized way while the electricity market features centralized clearing via the usual uniform-price auction. We assume that the natural gas market has unlimited supply, but may have constraints on transmission. Our natural gas system is divided into a series of interconnected zones that obey the spatial price arbitrage condition,

$$
p_{\text {downstream }}=p_{\text {upstream }}+t(q)+f(q . .)
$$

Here, $p_{\text {downstream }}$ and $p_{\text {upstream }}$ denotes the natural gas market zonal price for downstream zone and upstream zone respectively. $\mathrm{q}$ is the amount transferred from upstream zone to downstream zone. $t(q)$ is transmission cost, a fixed percentage of $q$. $f(q)$ is price difference function of downstream zone and upstream zone. If $q$ is smaller than transmission capacity limitation between these two zones, $f(q)=$ 0 . Otherwise, $f(q)$ is continuous monotone increasing (for simplicity we assume that $f(q)$ is linear. Also for simplicity we assume $p_{\text {upstream zone }}=0$, and assume no transmission cost.

\subsection{Players:}

Our model features two natural gas power-plant player types (with different heat rates and therefore marginal costs), a non-gas power plant player type, and a natural gas storage player. We do not explicitly model the electricity market operator as a strategic player. We assume one player of each type, but discuss relaxing this assumption in Section 2. The natural gas system is represented by a two-zone model, with a production zone and consumption zone (where the electricity market is located). We use $p_{g}$ indicate natural gas market zonal price and $p_{e}$ for power generation market clearing price in region.

- Player A: natural gas fired power plant with generation capacity $\bar{q}_{A}$. Marginal cost:

$$
M C_{A}=r_{A} p_{g}+c_{A}
$$

- Player B: natural gas fired power plant with generation capacity $\bar{q}_{B}$. Marginal cost:

$$
M C_{B}=r_{B} p_{g}+c_{B}
$$

- Player C: Power plant that uses a fuel other than natural gas and has a constant marginal cost of $\mathrm{C}$. The significance of this assumption is discussed further in Section 3.1.

- Player D: the only natural gas supplier in this zone, assume has unlimited capacity and ready to provide fuel to market. Assume player D has no operation cost and the marginal fuel cost is $\tilde{p}$.

Here, $r_{A}, c_{A}$ and $r_{B}, c_{B}$ are heat rate efficiency and fixed cost for player $\mathrm{A}$ and $\mathrm{B}$ respectively. Assume Player A and B have same generation capacity. $\bar{q}_{A}=$ $\bar{q}_{B}=\bar{q}$. Capacity limitation of player $\mathrm{C}$ is $\bar{q}_{C}$. Assume player $\mathrm{A}$ is less efficient than player $\mathrm{B}: r_{A}>r_{B}$. Thus, the natural gas that used by player $\mathrm{A}$ and player $\mathrm{B}$ for power generation are $r_{A} q_{A}$ and $r_{B} q_{B}$.

Let $q$ be the total amount of gas to be shipped into the downstream zone via natural gas transmission system before player D needs to supply gas to this zone. When player D provides $k$ amount of natural gas, the amount of natural gas has to be transferred into this zone is $q-k$ (holding total zonal demand constant), yielding a market price of $f(q-k)$.

All the players want to maximize their own payoffs in their relevant markets. Power plant profits are determined by the difference between the clearing price and the plant's marginal cost. Player D wants to optimize its natural gas supply amount $k^{*}$ satisfying equation (4) .

$$
\max _{k} p_{g} k=\max _{k} f(q-k) k
$$

Following Bushnell [12], we assume the natural gas inverse demand function is an exponential function, $f(q)=\exp (a q+b)$.

\subsection{Payoffs Under Different Cooperation Scenarios}

Here we describe the different potential modes of cooperation between Player D and the electricity market. We are concerned with the scenario when natural gas spot market price $p_{g}$ is at high level, so we can assume $p_{g}>C$. We outline four different cases.

Case 0: No coalition If power sector demand of natural gas is $q_{N G, e}, q_{N G, D}$ is all other demand besides 
power sector, then total demand in zone is $q_{N G, e}+$ $q_{N G, D}$. If player D won't provide any gas, all the gas has to be transferred via the gas transmission system, $q=q_{N G, e}+q_{N G, D}$. Let $k^{0}$ be the optimal supply amount of player D that satisfies equation (4), and the natural gas spot market price is $p_{g}^{0}=f\left(q-k^{0}\right)$.

If $p_{g}^{0}<C$, there is no incentive for player D to form a coalition with player $\mathrm{A}$ and/or player $\mathrm{B}$. In this case, player $\mathrm{C}$ is the marginal generator and player $\mathrm{D}$ has no incentive to adjust the amount of natural gas that it offers to the market (because players A and B are fully dispatched).

If $p_{g} \geq C$, player $\mathrm{A}$ has higher marginal cost than player $\mathrm{B}$, then player $\mathrm{A}$ is the marginal generator. $p_{e}^{0}=$ $M C_{A}^{0}$. Dispatched power generation is $\left\{q_{A}, \bar{q}_{B}, \bar{q}_{C}\right\}$. Since we have inelastic power demand, and $\bar{q}_{A}=\bar{q}_{B}=$ $\bar{q}$, let $\bar{q}-q_{A}=\Delta$, dispatched power generation can be written as $\left\{\bar{q}-\Delta, \bar{q}, \bar{q}_{C}\right\} . q_{e}=2 \bar{q}-\Delta+\bar{q}_{C}$. Thus, Fuel used by player A and player B: $\left\{r_{A}(\bar{q}-\Delta), r_{B} \bar{q}\right\}$ (table 1).

The payoffs in the case where $p_{g} \geq C$ would be:

$$
\begin{aligned}
& \pi_{A}^{0}=0 \\
& \pi_{B}^{0}=\left(p_{e}^{0}-M C_{B}^{0}\right) \bar{q} \\
& \pi_{C}^{0}=\left(p_{e}^{0}-C\right) q_{C}^{0} \\
& \pi_{D}^{0}=\left(p_{g}^{0}-\tilde{p}\right) k^{0}
\end{aligned}
$$

Case 1 Player A and $D$ form coalition $\{A \cup D\} \quad$ We now turn to the case where Players $A$ and D form a coalition to increase joint profits in the electricity and natural gas markets. In this and other cooperative cases, we focus only on the joint profits and not the distribution of profits between cooperative players. This would be determined by the relative bargaining strength of the players, which is a topic for further work and is not the main focus of this analysis. We examine two physical mechanisms for Player D to supply gas to Player A, and then show that these physical mechanisms are equivalent in terms of implications for joint profits.

Player D cannot supply player A directly In this case, Player D is not co-located with Player A but Player D can supply Player A through a shared gas transmission network. We model a cooperative agreement where Player D buys gas at a low off-peak price and releases it into the gas transmission system during an on-peak period. During this period, Player D agrees to sell gas directly to Player $A$ at the off-peak rate. This allows Player A to effectively under-bid in the electricity market by submitting an offer lower than $C$ (relative to the true marginal cost of Player A based on the market price of gas). Then, player A won't be the marginal generator (figure (2)). This reduces the profits of Player A but increases the profits of Player D, since overall gas demand increases. These profits are shared with Player A.

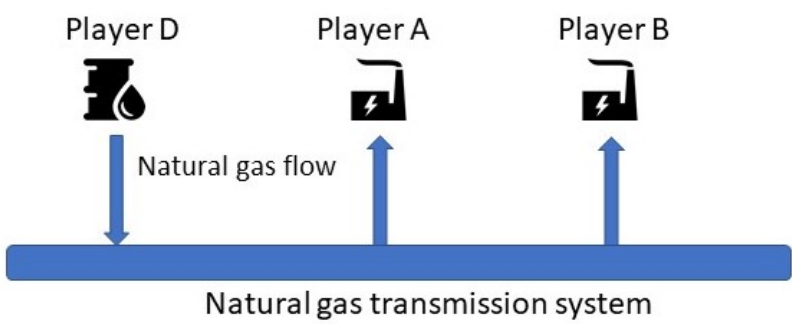

Figure 1. Player D can't supply player A directly

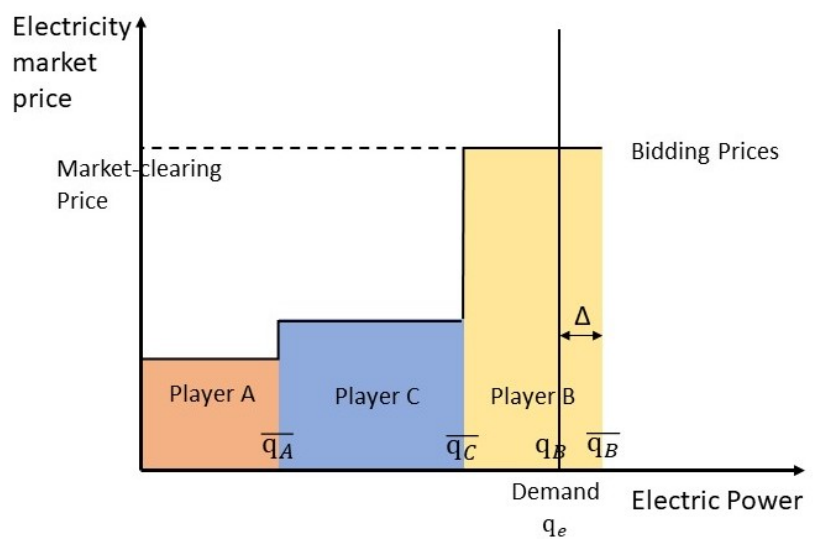

Figure 2. Case 1 Electric marketing clearing

As the result, player $\mathrm{B}$ is the marginal generator. $p_{e}^{1}=M C_{B}^{1}$. Dispatched power generation: $\left\{\bar{q}_{A}, q_{B}, \bar{q}_{C}\right\}=\left\{\bar{q}, \bar{q}-\Delta, \bar{q}_{C}\right\}$ (table 1). Fuel used by player $\mathrm{A}$ and $\mathrm{B}:\left\{r_{A} \bar{q}, r_{B}(\bar{q}-\Delta)\right\} . k^{1}$ is the optimal releasing amount of player $\mathrm{D}$ in this case, that satisfying equation (4). Natural gas market price is $p_{g}^{1}=f\left(q-k^{1}\right)$.

$$
\begin{aligned}
& \text { Marginal cost of player A: } M C_{A}^{1}=r_{A} p_{g}^{1}+c_{A} \\
& \text { Marginal cost of player B: } M C_{B}^{1}=r_{B} p_{g}^{1}+c_{B}
\end{aligned}
$$

The payoffs in this case would be:

$$
\begin{aligned}
\pi_{\{A \cup D\}}^{1} & =\underbrace{\left(p_{g}^{1} k^{1}-\tilde{p} k^{1}\right)}_{\text {profit on natural gas market }}+\underbrace{\left(p_{e}^{1}-M C_{A}^{1}\right) \bar{q}}_{\text {profit on electricity market }} \\
& =\left(p_{g}^{1}-\tilde{p}\right) k^{1}+\left(M C_{B}^{1}-M C_{A}^{1}\right) \bar{q} \\
\pi_{B}^{1} & =0 \\
\pi_{C}^{1} & =\left(p_{e}^{1}-C\right) \bar{q}_{C}=\left(M C_{B}^{1}-C\right) \bar{q}_{C}
\end{aligned}
$$


Player D can supply player A directly In this scenario, Players A and D are co-located. They may be offtakers in the same location from the gas transmission system, or Player D may be physically co-located with Player A, as in on-site natural gas storage.

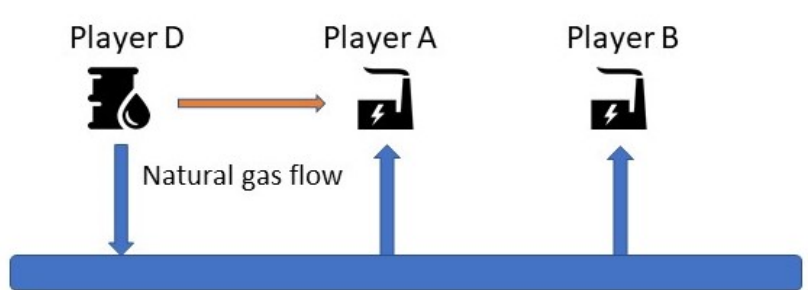

Natural gas transmission system

Figure 3. Player D can supply player A directly

Thus the marginal fuel cost of player $\mathrm{A}$ is $\tilde{p}$, the fuel cost of player D. The impact of this physical arrangement is that Player D can acquire natural gas at a low price during the off-peak gas period, and supply Player A during the on-peak gas period. This affects the overall demand for gas in the gas consumption zone. The payoff of this coalition is the same as if Players A and $\mathrm{D}$ did not share any physical connection, as we show below:

$$
\begin{aligned}
& \text { Marginal cost of player A: } M C^{1^{\prime}}{ }_{A}=r_{A} \tilde{p}+c_{A} \\
& \text { Marginal cost of player B: } M C^{1^{\prime}}{ }_{B}=r_{B} p_{g}^{1}+c_{B}=M C_{B}^{1}
\end{aligned}
$$

$$
\begin{aligned}
\pi_{\{A \cup D\}}^{1^{\prime}}= & \underbrace{\left(p_{g}^{1}-\tilde{p}\right)\left(k^{1}-r_{A} q_{A}^{1}\right)}_{\text {profit on natural gas market }} \\
& +\underbrace{\left(p_{e}^{1}-M C^{1^{\prime}}\right) q_{A}^{1}}_{\text {profit on electricity market }} \\
= & p_{g}^{1} k^{1}-\tilde{p} k^{1}-r_{A} p_{g}^{1} \bar{q}+M C_{B}^{1} \bar{q}-c_{A} \bar{q} \\
= & \left(p_{g}^{1}-\tilde{p}\right) k^{1}+\left(M C_{B}^{1}-r_{A} p_{g}^{1} \bar{q}-c_{A}\right) \bar{q} \\
= & \left(p_{g}^{1}-\tilde{p}\right) k^{1}+\left(M C_{B}^{1}-M C_{A}^{1}\right) \bar{q} \\
\Longrightarrow & \pi_{\{A \cup D\}}=\pi_{\{A \cup D\}}^{\prime}
\end{aligned}
$$

Value of coalition $\{A \cup D\} \quad$ In case 0 , power sector consumption of natural gas is:

$$
q_{N G, e}^{0}=r_{A}(\bar{q}-\Delta)+r_{B} \bar{q}
$$

In case 1 , this consumption is:

$$
q_{N G, e}^{1}=r_{A} \bar{q}+r_{B}(\bar{q}-\Delta)=q_{N G, e}^{0}+\left(r_{A}-r_{B}\right) \Delta
$$

Since non-power plant natural gas demand $q_{N G, d}$ is inelastic and $r_{A}>r_{B}$, we have total natural gas demand

$$
q_{N G}^{0}=q_{N G, d}+q_{N G, e}^{0}<q_{N G, d}+q_{N G, e}^{1}=q_{N G}^{1}
$$
$p_{g}^{1}$

As shown earlier, we would have $k^{0} \leq k^{1}$ and $p_{g}^{0} \leq$

To calculate $V_{\{A \cup D\}}$, we compare $\pi_{G}^{1}$, the increase in gas market profits, with $\pi_{P}^{1}$, the loss on power market profits.

We assume when there is no any coalition formed (case 0$)$,

$$
M C_{A}^{0}-\epsilon=M C_{B}^{0}=C \quad \epsilon>0
$$

Since $p_{g}^{0} \leq p_{g}^{1}$ and $r_{A}>r_{B}$

$$
M C_{A}^{1}>M C_{B}^{1} \geq C
$$

Equality in (22) holds only when $\Delta$ is not large enough. $\epsilon$ is a critical parameter here. It indicates the relationship of marginal costs between player A, B and $C$. In practice, this value should be straightforward to calculate, so we can use it to derive a condition on whether $A \cup D$ cooperation has any value.

We use the relationship in (22) to rewrite payoffs as follows. For the purpose of this exposition, we will assume a linear form for the inverse demand curve $p_{g}=f(q)=a q+b$. We note, however, that a similar property holds in the case of an exponential inverse demand curve.

- Case 0:

$$
\begin{aligned}
& \pi_{A}^{0}=0 \\
& \pi_{B}^{0}=\bar{q}_{B} \epsilon \\
& \pi_{C}^{0}=0 \\
& \pi_{D}^{0}=k^{0}\left(p_{g}^{0}-\tilde{p}\right) \\
& \pi_{A}^{0}+\pi_{D}^{0}=k^{0}\left(p_{g}^{0}-\tilde{p}\right)
\end{aligned}
$$

- Case 1:

$$
\begin{aligned}
& \pi_{G}^{1}=\pi_{D}^{1}-\pi_{D}^{0} \\
& \pi_{P}^{1}=\pi_{A}^{1}-\pi_{A}^{0}
\end{aligned}
$$

Noting that for profit maximization we would have $k^{*}=$ $\frac{a q_{N G}+b}{2 a}$, and inserting the linear demand curve, we then have: 


$$
\begin{aligned}
k^{0} & =\frac{1}{2}\left(q_{N G}^{0}+\frac{b}{a}\right) \\
p_{g}^{0} & =a\left(q_{N G}^{0}-k^{0}\right)+b=\frac{a}{2}\left(q_{N G}^{0}+\frac{b}{a}\right) \\
k^{1} & =\frac{1}{2}\left(q_{N G}^{0}+\Delta\left(r_{A}-r_{B}\right)+\frac{b}{a}\right) \\
& =k^{0}+\frac{1}{2} \Delta\left(r_{A}-r_{B}\right) \\
p_{g}^{1} & =a\left(q_{N G}^{1}-k^{1}\right)+b \\
& =a\left(q_{N G}^{0}-k^{0}\right)+b+\frac{1}{2} a \Delta\left(r_{A}-r_{B}\right) \\
& =p_{g}^{0}+\frac{1}{2} a \Delta\left(r_{A}-r_{B}\right)
\end{aligned}
$$

$$
\begin{aligned}
M C_{A}^{1} & =r_{A}\left(p_{g}^{0}+\frac{1}{2} a \Delta\left(r_{A}+r_{B}\right)\right)+c_{A} \\
& =(C+\epsilon)+\frac{1}{2} a \Delta\left(r_{A}+r_{B}\right) \\
M C_{B}^{1} & =r_{B}\left(p_{g}^{0}+\frac{1}{2} a \Delta\left(r_{A}+r_{B}\right)\right)+c_{B} \\
& =C+\frac{1}{2} a \Delta r_{B}\left(r_{A}-r_{B}\right)
\end{aligned}
$$

$$
\begin{aligned}
\Longrightarrow \pi_{G}^{1}= & k^{1}\left(p_{g}^{1}-\tilde{p}\right)-k^{0}\left(p_{g}^{0}-\tilde{p}\right) \\
= & \left(\frac{1}{2} \Delta\left(r_{A}-r_{B}\right)+k^{0}\right)\left(p_{g}^{0}+\frac{1}{2} a \Delta\left(r_{A}-r_{B}\right)\right. \\
& -\tilde{p})-k^{0}\left(p_{g}^{0}-\tilde{p}\right) \\
= & \frac{1}{2} a \Delta\left(r_{A}-r_{B}\right)\left(q_{N G}^{0}+\frac{b}{a}-\frac{\tilde{p}}{a}\right) \\
& +\frac{1}{4} a \Delta^{2}\left(r_{A}-r_{B}\right)^{2} \\
\pi_{P}^{1}= & \left(M C_{B}^{1}-M C_{A}^{1}\right) \bar{q}_{A} \\
= & {\left[\left(C+\frac{1}{2} a \Delta\left(r_{A}-r_{B}\right) r_{B}\right)-(C+\epsilon\right.} \\
& \left.\left.+\frac{1}{2} a \Delta\left(r_{A}-r_{B}\right) r_{A}\right)\right] \bar{q}_{A} \\
= & -\epsilon \bar{q}_{A}
\end{aligned}
$$

$$
\begin{aligned}
& \text { If } V_{\{A \cup D\}} \geq 0 \text {, } \\
& \pi_{G}^{1}+\pi_{P}^{1} \geq 0 \\
& \Longrightarrow \frac{1}{2} a \Delta\left(r_{A}-r_{B}\right)\left(q_{N G}^{0}+\frac{b}{a}-\frac{\tilde{p}}{a}\right) \\
& +\frac{1}{4} a \Delta^{2}\left(r_{A}-r_{B}\right)^{2}-\epsilon \bar{q}_{A} \geq 0 \\
& \Longrightarrow \epsilon \leq \frac{\frac{1}{2} a \Delta\left(r_{A}-r_{B}\right)\left[q_{N G}^{0}+\frac{b}{a}-\frac{\tilde{p}}{a}+\frac{1}{2} \Delta\left(r_{A}-r_{B}\right)\right]}{\bar{q}_{A}} \\
& \Longrightarrow 0<\epsilon \leq \frac{1}{2 \bar{q}_{A}} a \Delta\left(r_{A}-r_{B}\right)\left[\left(q_{N G, D}+\bar{q}_{N G, e}\right)-\right. \\
& \left.\frac{1}{2} \Delta\left(r_{A}-r_{B}\right)+\frac{b}{a}-\frac{\tilde{p}}{a}\right]
\end{aligned}
$$

Under assumption (21), when $\Delta$ is too small or $\tilde{p}$ is too great, there may be no value of $\epsilon$ satisfying equation (40). In that case, $V_{\{A \cup D\}} \leq 0$ and neither player A or $\mathrm{D}$ would have incentive for cooperation.

Case 2: Player $B$ and $D$ form coalition $\{B \cup D\}$ Since player B is more efficient than player $\mathrm{A}$, the coalition strategy defined for $\{A \cup D\}$ won't change the dispatch order relative to the case of no coalition. There would thus be no gain for B or D to cooperate.

Case 3 Player $\mathbf{A}, \mathbf{B}$ and $\mathbf{D}$ form coalition $\{A \cup B \cup D\}$ The final case that we consider is for Players $\mathrm{A}$ and $\mathrm{B}$ to jointly form a coalition with Player D. The strategy for this coalition would be similar to the $\{A \cup D\}$ coalition - Players A and B would submit offers to the power market operator below actual marginal costs. This pushes Player $\mathrm{C}$ to the margin in the power market (figure (4)). The extra profits in the natural gas market would be shared between Players A, B and D.

The dispatch in this case is $\left\{\bar{q}_{A}, \bar{q}_{B}, q_{C}^{3}\right\}=$ $\left\{\bar{q}_{A}, \bar{q}_{B}, \bar{q}_{C}-\Delta\right\}=\left\{q_{A}^{0}+\Delta, \bar{q}_{B}, \bar{q}_{C}-\Delta\right\}$. Total power sector usage of natural gas is $r_{A} \bar{q}_{A}+r_{B} \bar{q}_{B}$. Natural gas power plants in case 3 get dispatched at a higher level than in case 0 , by an amount $\Delta$. Natural gas total demand in region increases by $r_{A} \Delta$ compared to case 0 (table (1)), so we would have

$$
q_{N G}^{3}=q_{N G}^{0}+\Delta r_{A}
$$

Thus, as we claimed before, optimal value $k^{3}>$ $k^{1}>k^{0}, p_{g}^{3}>p_{g}^{1}>p_{g}^{0}$. Since the payoff of coalition $\{A \cup B \cup D\}$ is invariant to the physical 


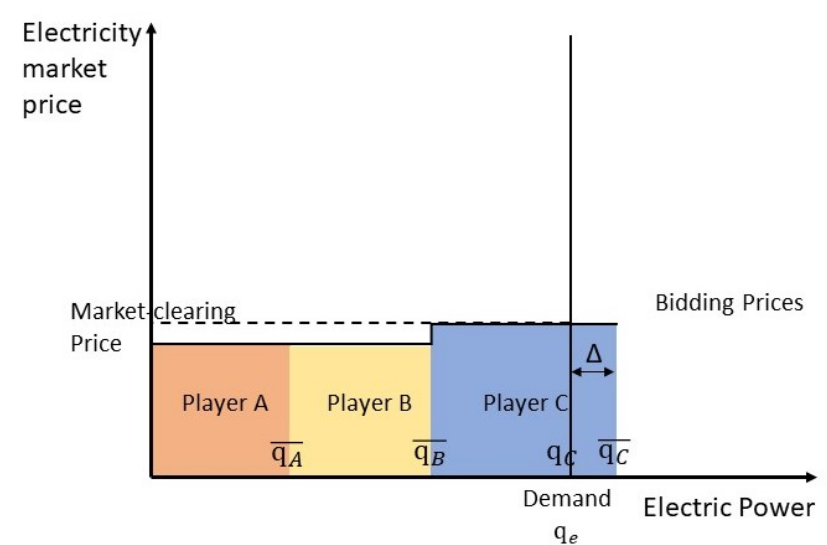

Figure 4. Case 3 Electric marketing clearing

interconnection mechanism between Player D and the gas-fired generators (as we saw in case 1), we focus on the scenario where player D injects gas into the transmission system directly.

Generator marginal costs in this case are given by:

$$
\begin{aligned}
& M C_{A}^{3}=r_{A} p_{g}^{3}+c_{A} \\
& M C_{B}^{3}=r_{B} p_{g}^{3}+c_{B} \\
& M C_{C}=C
\end{aligned}
$$

Since Player $\mathrm{C}$ is the marginal generator, we also have $p_{e}^{3}=C$. The payoffs can be written as:

$$
\begin{aligned}
\pi_{C}^{3}= & 0 \\
\pi_{\{A \cup B \cup D\}}= & \left(C-M C_{A}^{3}\right) \bar{q}_{A}+\left(C-M C_{B}^{3}\right) \bar{q}_{B} \\
& +\left(p_{g}^{3}-\tilde{p}\right) k^{3}
\end{aligned}
$$

The payoff condition for the coalition can be rewritten as:

$$
\begin{aligned}
\pi_{\{A \cup B \cup D\}}= & \underbrace{\left[-\epsilon \bar{q}_{A}-\bar{q}_{A} r_{A}\left(p_{g}^{3}-p_{g}^{0}\right)\right]}_{\text {profits from A }} \\
& +\underbrace{\left[-\bar{q}_{B} r_{B}\left(p_{g}^{3}-p_{g}^{0}\right)\right]}_{\text {profits from B }} \\
& +\underbrace{\left[k^{3}\left(p_{g}^{3}-\tilde{p}\right)\right]}_{\text {profits from D }}
\end{aligned}
$$

Here, $k^{3}$ satisfies $\max _{k^{3}} p_{g}^{3} k^{3}$.

Again, if we assume a linear inverse demand curve for natural gas, we can derive an analogous condition on $\epsilon$ for the value of coalition $\{A \cup B \cup D\}$ as we did for
$\{A \cup D\}$

$$
\begin{aligned}
k^{3}= & \frac{1}{2}\left(q_{N G}^{0}+\Delta r_{A}+\frac{b}{a}\right)=k^{0}+\frac{1}{2} \Delta r_{A} \\
p_{g}^{3}= & a\left(q_{N G}^{3}-k^{3}\right)+b \\
= & a\left(q_{N G}^{0}-k^{0}\right)+b+\frac{1}{2} a \Delta r_{A} \\
= & p_{g}^{0}+\frac{1}{2} a \Delta r_{A} \\
\Longrightarrow k^{3}\left(p_{g}^{3}-\tilde{p}\right)= & \left(\frac{1}{2} \Delta r_{A}+k^{0}\right)\left(p_{g}^{0}+\frac{1}{2} a \Delta r_{A}-\tilde{p}\right) \\
= & k^{0}\left(p_{g}^{0}-\tilde{p}\right)+\left[\frac{1}{2} \Delta r_{A}\left(p_{g}^{0}-\tilde{p}\right)\right. \\
& \left.+\frac{1}{4} a \Delta^{2} r_{A}^{2}+\frac{1}{2} a \Delta r_{A} k^{0}\right]
\end{aligned}
$$

To evaluate if there is extra value associated with the coalition $\{A \cup B \cup D\}$ as compared with case 0 (no coalition), we compare the increase in natural gas market profits with the loss in power market profits.

Lost power market profits:

$\pi_{P}^{3}=-\left[\epsilon \bar{q}_{A}+\bar{q}_{A} r_{A}\left(p_{g}^{3}-p_{g}^{0}\right)+\bar{q}_{B} r_{B}\left(p_{g}^{3}-p_{g}^{0}\right)+\epsilon \bar{q}_{B}\right]$

Increased natural gas market profits:

$$
\pi_{G}^{3}=\frac{\Delta}{2} r_{A}\left(p_{g}^{0}-\tilde{p}\right)+\frac{\Delta}{2} a r_{A} k^{0}+\frac{\Delta^{2}}{4} a r_{A}^{2}
$$

Substitute (30) (31) (48) and (49) into (51) and (52):

$$
\begin{gathered}
\pi_{P}^{3}=-\left[\frac{\Delta}{2} a r_{A}\left(r_{A} \bar{q}_{A}+r_{B} \bar{q}_{B}\right)+\epsilon\left(\bar{q}_{A}+\bar{q}_{B}\right)\right] \\
\pi_{G}^{3}=\frac{a}{2}\left(\Delta r_{A}\right)\left(q_{N G}^{0}+\frac{b}{a}-\frac{\tilde{p}}{a}\right)+\frac{\Delta^{2}}{4} a r_{A}^{2}
\end{gathered}
$$

We want $V_{\{A \cup B \cup D\}} \geq 0$, so we evaluate:

$$
\begin{aligned}
V_{\{A \cup B \cup D\}}= & \pi_{G}+\pi_{P} \\
= & \frac{a}{2} \Delta r_{A}\left(q_{N G}^{0}+\frac{b}{a}-\frac{\tilde{p}}{a}-r_{A} \bar{q}_{A}-r_{B} \bar{q}_{B}\right) \\
& +\frac{\Delta^{2}}{4} a r_{A}^{2}-\epsilon\left(\bar{q}_{A}+\bar{q}_{B}\right) \geq 0
\end{aligned}
$$




$$
\begin{aligned}
\Longrightarrow \epsilon\left(\bar{q}_{A}+\bar{q}_{B}\right) & \leq \frac{a}{2} \Delta r_{A}\left(q_{N G}^{0}+\frac{b}{a}-\frac{\tilde{p}}{a}\right) \\
& -\frac{a}{2} \Delta r_{A}\left(r_{A} \bar{q}_{A}+r_{B} \bar{q}_{B}\right) \\
& =\frac{a}{2} \Delta r_{A}\left(q_{N G}^{0}-\left(r_{A} \bar{q}_{A}+r_{B} \bar{q}_{B}\right)\right. \\
& \left.+\frac{b}{a}-\frac{\tilde{p}}{a}\right) \\
& =\frac{a}{2} \Delta r_{A}\left[\left(q_{N G, d}+\left(\bar{q}_{N G, e}-r_{A} \Delta\right)\right)\right. \\
& \left.-\bar{q}_{N G, e}+\frac{b}{a}-\frac{\tilde{p}}{a}\right] \\
& =\frac{a}{2} \Delta r_{A}\left(q_{N G, d}-r_{A} \Delta+\frac{b}{a}-\frac{\tilde{p}}{a}\right) \\
\Longrightarrow & \leq \frac{\frac{a}{2} \Delta r_{A}\left(q_{N G, d}-r_{A} \Delta+\frac{b}{a}-\frac{\tilde{p}}{a}\right)}{\bar{q}_{e}}
\end{aligned}
$$

Here, $q_{N G, d}$ is all the natural gas demand except power section demand. $\bar{q}_{e}$ is the total capacity of all natural gas fired power plants (player A and B). $\bar{q}_{N G, e}$ is the fuel usage when $\bar{q}_{e}$ amount of power generation is dispatched to natural gas fired generators. $\Delta$ describes how much more power natural gas fired power plants can get via cooperating with player $\mathrm{D}$.

Therefore, when $\epsilon$, the marginal cost difference with player $\mathrm{A}$ (the one with highest marginal cost in coalition) and $\mathrm{C}$, is smaller than the threshold derived above, there is extra value for coalition.

When player $\mathrm{C}$ is the marginal generator in case 0 , $\Delta=0$, and $V_{\{A \cup B \cup D\}}=0$. When $M C_{A}^{0}$ or $M C_{B}^{0}>$ $C$, marginal generator is natural gas fired power plant, $\Delta>0$ and coalition $\{A \cup B \cup D\}$ can have non-zero value.

Table 1 summarizes the four cases that we have considered for this cooperative static game.

A potentially interesting extension of this framework would be to assume multiple actors in the market for each player type. This extension is beyond the scope of the present paper, but we provide some intuition as to how the model's results may be impacted for each of the three cases if there are multiple actors of firm types A and B. In case 1, suppose that only one of the type A players forms a coalition with player D. Recall from the discussion of case 1 that the driver of value for the $A \cup D$ coalition is the increased profits in the natural gas market. These profits will increase as more actors join the coalition, and one actor of type A cannot free-ride on another actor of type A. Thus, the Nash equilibrium would be for all type A players to join the coalition.
A similar situation arises for case 3, where the Nash equilibrium is for all actors of types $A$ and $B$ to join the coalition (or for none to join the coalition, depending on the values of the parameters in equation (57)). Since there is no extra value for the coalition in case 2 , we do not need to consider this case any further.

\section{Illustrative Examples and Sensitivities}

We illustrate our cooperative framework using some realistic parameters from the natural gas market. Our natural gas prices for the gas consumption zone are based on Transco Zone 6 New York sub-zone data( from Janunary 2012 to June 2014, access from SNL) - We divide all natural gas fired power plants in this region into two player types. Player A has lower heat rate efficiency, with average heat rate 9.142 $m m B T U / M W h$. Player B has higher efficiency with average heat rate $6.431 \mathrm{mmBTU} / M W h$.

Marginal costs for Players A and B are given below; we continue to assume that Player $\mathrm{C}$ has constant marginal cost $C$.

$$
\begin{aligned}
& \text { Player A: } M C_{A}=r_{A} p_{g}+c_{A}=9.142 p_{g}+24 \\
& \text { Player B: } M C_{B}=r_{B} p_{g}+c_{B}=6.431 p_{g}+40
\end{aligned}
$$

Based on EIA 2014 natural gas consumption data [18], approximately $1 / 3$ of natural gas consumption in New York is used for electric power. We take $1 / 3$ of natural gas consumption in zone as the total capacity of player A and $\mathrm{B}$, and assume $\bar{q}_{A}=\bar{q}_{B}$. We assume player $\mathrm{D}$ is a natural gas supplier in this region with marginal cost $\tilde{p}$. The capacity of player $\mathrm{D}$ is unlimited. If output from Player $\mathrm{D}$ is zero, then the prevailing spot gas price is $p_{g}$.

Figure 5 shows the value of the coalitions $\{A \cup D\}$ and $\{A \cup B \cup D\}$ as a function of the spot price of natural gas (without any coalition) in the consumption zone. When natural gas market price is high enough that player $\mathrm{A}$ is the marginal generator, the value of coalition $\{A \cup D\}$ is always positive, and increases with the price of natural gas. As we showed earlier, positive value of coalition $\{A \cup B \cup D\}$ only exists in a small range (equation (57)). Interestingly, at very high levels of the natural gas price (greater than $\$ 22$ per million BTU) the value of coalition $\{A \cup B \cup D\}$ becomes negative while the value of coalition $\{A \cup D\}$ remain high. The intuition here is that because Player B would have been on the margin (at a high electricity market price) in the absence of any cooperative behavior, the profits of coalition $\{A \cup B \cup D\}$ in natural gas market can not cover the loss in the electricity market. 
Table 1. Case Comparison

\begin{tabular}{|c|c|c|c|c|c|}
\hline & & case 0 & & & \\
\hline Player & & $\mathrm{A}$ & B & $\mathrm{C}$ & $\mathrm{D}$ \\
\hline Marginal Cost & & $r_{A} p_{A}^{0}+c_{A}$ & $r_{B} p_{B}^{0}+c_{B}$ & $\mathrm{C}$ & $\tilde{p}$ \\
\hline Marginal Generator & & $\checkmark($ as assumed $)$ & & & \\
\hline Dispatched Power Ge & neration & $q_{A}$ & $\bar{q}_{B}$ & $\bar{q}_{C}$ & \\
\hline Natural Gas Usage & $\begin{array}{l}\text { By Generator } \\
\text { In Reoion }\end{array}$ & $r_{A} q_{A}$ & $r_{B} \bar{q}_{B}$ & & $-k^{0}$ \\
\hline Profit & & $\begin{array}{l}q_{N G}-\varphi_{N G}, \\
0\end{array}$ & $\left(M C_{A}^{0}-M C_{B}^{0}\right) \bar{q}_{B}$ & $\left(M C_{A}^{0}-C\right) \bar{q}_{C}$ & $\left(p_{g}^{0}-\tilde{p}\right) k^{0}$ \\
\hline & & case 1 & & & \\
\hline Player & & $\mathrm{A}$ & $\mathrm{B}$ & $\mathrm{C}$ & $\mathrm{D}$ \\
\hline $\begin{array}{l}\text { Marginal Cost } \\
\text { Marginal Generator }\end{array}$ & & $r_{A} p_{A}^{1}+c_{A}$ & $r_{B} p_{B}^{1}+c_{B}$ & $\mathrm{C}$ & $\tilde{p}$ \\
\hline Dispatched Power Ge & neration & & & $\bar{q}_{C}$ & \\
\hline Natural Gas Usage & $\begin{array}{l}\text { By Generator } \\
\text { In Region }\end{array}$ & $\begin{array}{l}r_{A}\left(q_{A}+\Delta\right) \\
q^{0}+\left(r_{A}+r_{B}\right) \Delta\end{array}$ & $r_{B}\left(\bar{q}_{B}-\Delta\right)$ & & $-k^{1}$ \\
\hline Profit & & $\left(M C_{B}^{1}-M C_{A}^{1}\right) \bar{q}_{A}$ & 0 & $\left(M C_{A}^{1}-C\right) \bar{q}_{C}$ & $\left(p_{g}^{1}-\tilde{p}\right) k^{1}$ \\
\hline & & case 2 & & & \\
\hline Player & & $\mathrm{A}$ & $\mathrm{B}$ & $\mathrm{C}$ & $\mathrm{D}$ \\
\hline $\begin{array}{l}\text { Marginal Cost } \\
\text { Marginal Generator }\end{array}$ & & $r_{A} p_{A}^{2}+c_{A}$ & $r_{B} p_{B}^{2}+c_{B}$ & $\mathrm{C}$ & $\tilde{p}$ \\
\hline Dispatched Power Ge & neration & $q_{A}$ & $\bar{q}_{B}$ & $\bar{q}_{C}$ & \\
\hline Natural Gas Usage & $\begin{array}{l}\text { By Generator } \\
\text { In Region }\end{array}$ & $\begin{array}{l}r_{A A} q_{A} \\
q_{N G}^{0}\end{array}$ & $r_{B} \bar{q}_{B}$ & & $-k^{2}$ \\
\hline Profit & & 0 & $\left(M C_{A}^{2}-M C_{B}^{2}\right) \bar{q}_{B}$ & $\left(M C_{A}^{2}-C\right) \bar{q}_{C}$ & $\left(p_{g}^{2}-\tilde{p}\right) k^{2}$ \\
\hline & & case 3 & & & \\
\hline Player & & $\mathrm{A}$ & $\mathrm{B}$ & $\mathrm{C}$ & $\mathrm{D}$ \\
\hline $\begin{array}{l}\text { Marginal Cost } \\
\text { Marginal Generator }\end{array}$ & & $r_{A} p_{A}^{3}+c_{A}$ & $r_{B} p_{B}^{3}+c_{B}$ & $\begin{array}{l}\mathrm{C} \\
\checkmark\end{array}$ & $\tilde{p}$ \\
\hline Dispatched Power Ge & neration & $\bar{q}_{A}$ & $\bar{q}_{B}$ & $\bar{q}_{C}$ & \\
\hline Natural Gas Usage & $\begin{array}{l}\text { By Generator } \\
\text { In Region }\end{array}$ & $\begin{array}{l}r_{A} \bar{q}_{A} \\
q_{N G}^{0}+r_{A} \Delta\end{array}$ & $r_{B} \bar{q}_{B}$ & & $-k^{3}$ \\
\hline Profit & & $\left(C-M C_{A}^{3}\right) \bar{q}_{A}$ & $\left(C-M C_{B}^{0}\right) \bar{q}_{B}$ & 0 & $\left(p_{g}^{3}-\tilde{p}\right) k^{3}$ \\
\hline
\end{tabular}

\subsection{Sensitivity analysis}

To better understand the drivers of the value of coalitions $\{A \cup D\}$ and $\{A \cup B \cup D\}$, we perform a sensitivity analysis of the coalition value on several model parameters: $\left(\tilde{p}, C\right.$ and $\left.\frac{\Delta}{\bar{q}}\right)$. In each of these simulations we vary one of these parameters and the natural gas price, and observe how the value of the coalitions $\{A \cup D\}$ and $\{A \cup B \cup D\}$ change. These sensitivity results are shown in Figures (6), (7) and (??).

In each plot, there are multiple panels. The number on the top of each panel is the value of the sensitivity parameter setup for that panel. In each panel in each plot, the natural gas market price (without player D in the market) is on the horizontal axis. The vertical axis measures the value of the coalition. "A+B+D" means coalition $\{A \cup B \cup D\}$, and "A+D" indicates coalition $\{A \cup D\}$. We note that the scale for the vertical axis is not consistent between figures (but is consistent between panels in a single figure).

Referring to Figure 6, we observe two behaviors of interest in the value of the $\{A \cup D\}$ coalition as $\tilde{p}$ goes from $0 \$ / \mathrm{mmBTU}$ to $5 \$ / \mathrm{mmBTU}$. First, overall the value of the coalition declines as $\tilde{p}$ increases. Second, the value of the natural gas price at which the value of the coalition rises above zero decreases as $\tilde{p}$ increases, although once for values of $\tilde{p}$ equal to $\$ 4$ or $\$ 5$ per million BTU the value of $\{A \cup D\}$ varies very little from zero. The value of $\{A \cup B \cup D\}$ is positive only for small values of $\tilde{p}$ and at moderate gas prices.

The influence of $C$ on the value of coalition is a little bit different from $\tilde{p}$. In the first panel of figure (7), $C=70 \$ / \mathrm{mmBTU}$, we observe that the value of $\{A \cup B \cup D\}$ exceeds the value of $\{A \cup D\}$ for some lower gas price values. This is because of the behavior of the marginal cost curves of the power plants. When $p_{g}$ is low, the marginal generator is player $\mathrm{C}$ in the absence of a coalition. As $p_{g}$ increases, the marginal costs of player A and B are also increasing; in particular the marginal cost of $\mathrm{A}$ increases faster than the marginal cost of $\mathrm{B}$ as gas prices rise. For lower gas prices and smaller values of $C$, Player $\mathrm{B}$ is on the margin and 


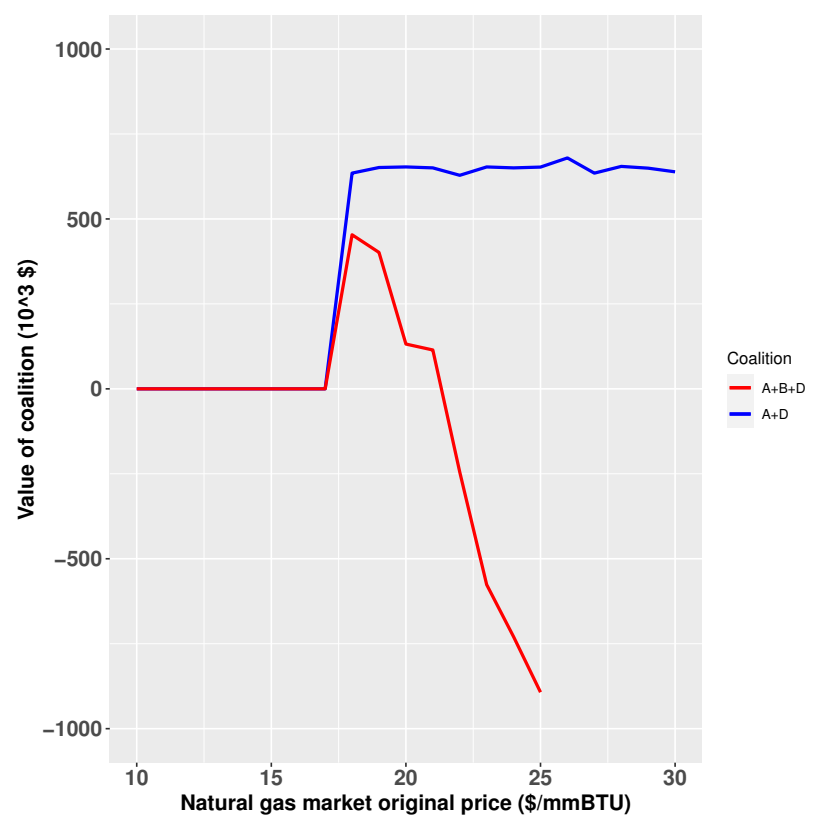

Figure 5. Value of Coalition

coalition $\{A \cup B \cup D\}$ has higher value. As the gas price rises, Player A moves to the margin and coalition $\{A \cup D\}$ has higher value. Higher values of $C$ change the range and magnitude of the amount by which the $\{A \cup B \cup D\}$ is more profitable.

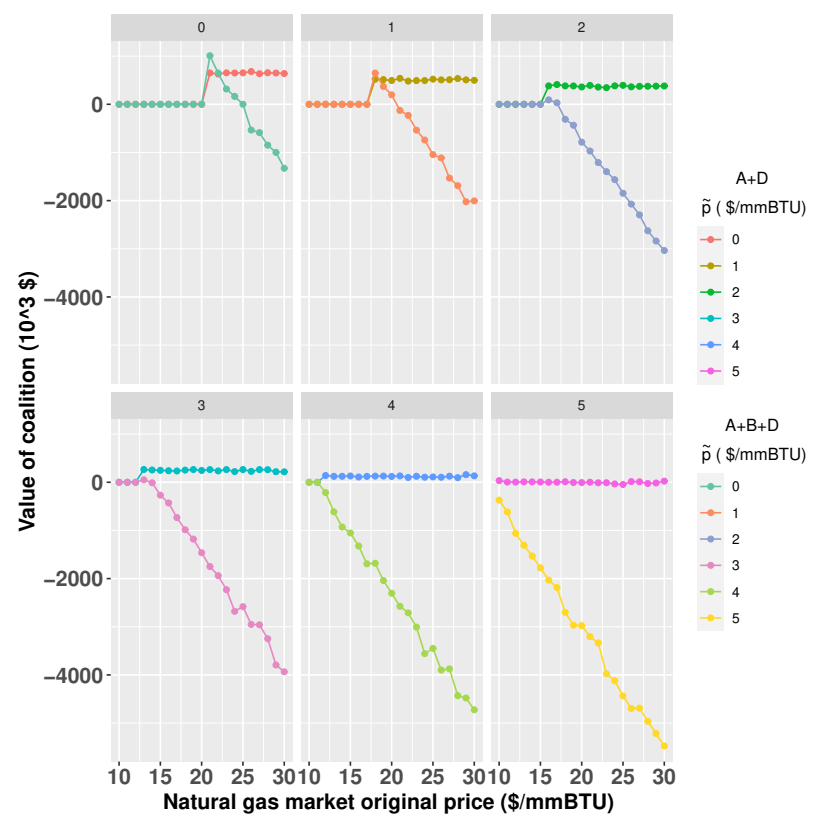

Figure 6. Value of coalition as $\tilde{p}$ varies

The assumption that player $\mathrm{C}$ has constant marginal

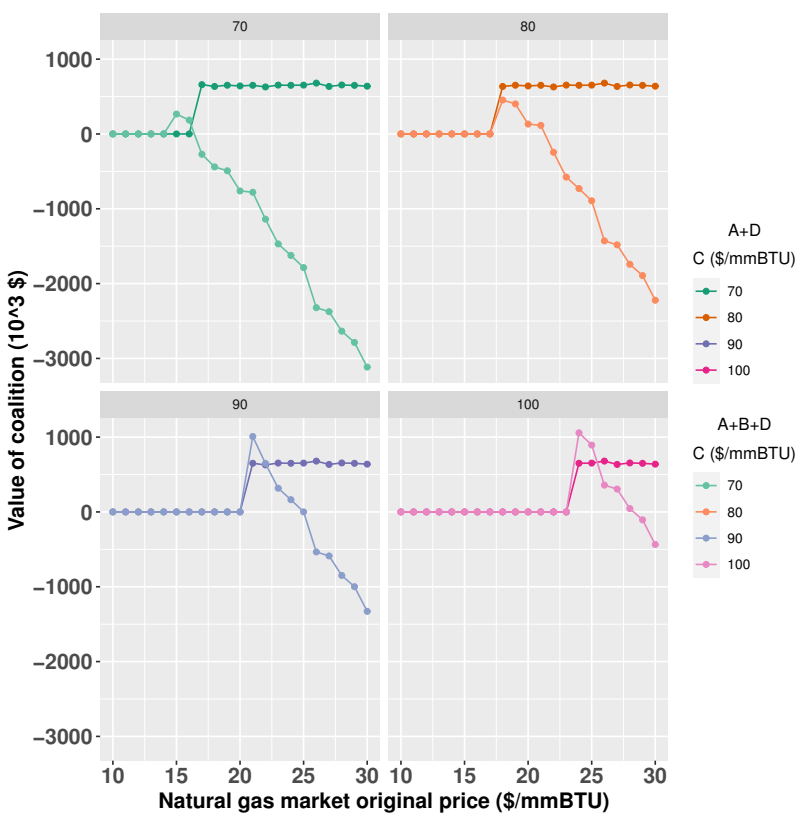

Figure 7. Value of coalition as $C$ varies

cost is a simplifying assumption, though the implication of this assumption depends on which case is considered. In cases 1 and 2, if the marginal cost of player $C$ is not constant, the coalition between player D and player A or B will not affect the dispatch point for player C. In case 3, the optimal coalition strategy will depend on how sensitive the marginal cost of player $\mathrm{C}$ is to the fuel usage. Figure (8) and (7) illustrates the relationship between the value of coalition $\{A \cup B \cup D\}$ and the marginal cost of player $C$ when this marginal cost is assumed constant. With non-constant marginal cost, the domain of the profitable region for the coalition will change.

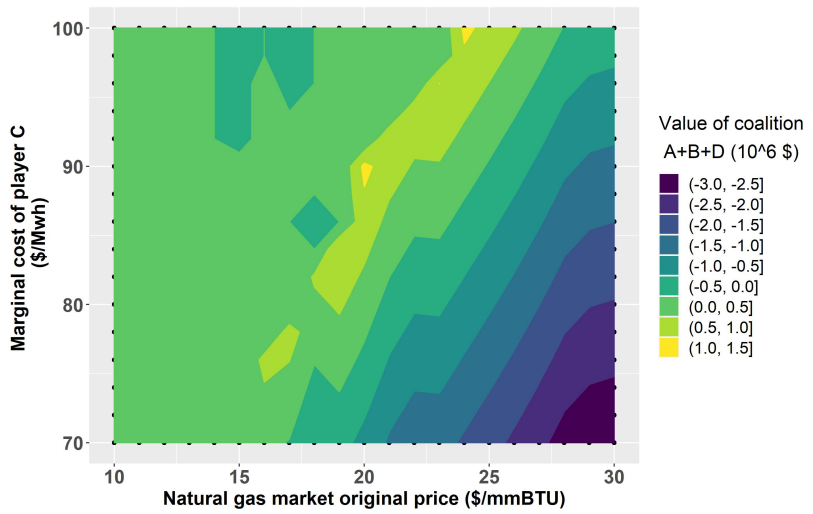

Figure 8. Value of coalition $\{A \cup B \cup D\}$ for Natural gas original market price and Marginal cost of player $C$ 


\section{Conclusion}

In a simple static cooperative game context, we find the potential for collusive behavior between gas storage units and gas-fired power plants operating in centralized electricity markets. Joint gas-electric profits can be increased in some circumstances through a strategy of temporal price arbitrage in the natural gas market and the manipulation of electric energy market prices through under-bidding. Whether this strategy yields any additional cross-market profits is highly sensitive to a number of different market parameters, and therefore collusive behavior will not have strategic value in all states of the market. We find that the value of the gas-grid coalition is particularly sensitive to the on-peak price of natural gas and the marginal cost of generator(s) participating in the coalition.

The present paper is limited to a static game context in a stylized electricity market, and additional research is needed to formulate specific policy prescriptions. The value of the gas-grid coalition declines as the number of participants grows, so monitoring and detection activities should focus more on smaller coalitions than larger coalitions. Importantly, we show that the potential for collusive behavior is independent of the physical mechanism of interconnection between the gas unit and the gas-fired power plant. Prohibiting or limiting vertical integration between gas storage and power plants, for example through co-location of storage with gas-fired plants, does not necessarily remove the potential for collusive behavior. Our modeling framework points towards the need for developing cross-market monitoring mechanisms to ensure that investments made to promote fuel security in electricity markets do not have deleterious unintended competitive consequences.

\section{Acknowledgements}

The authors acknowledge support from the U.S. National Science Foundation under award No. 1638331.

\section{References}

[1] S. Blumsack, "Should natural gas be shipped or stored to supply power plants?," in Proceedings of the 52nd Hawaii International Conference on System Sciences, 2019.

[2] C. Borraz-Sanchez and R. Bent, "Joint expansion planning for natural gas and electric transmission with endogenous market feedbacks," in Hawaii International Conference On System Sciences (HICSS-51), 2018.

[3] R. D. Tabors and S. Adamson, "Measurement of energy market inefficiencies in the coordination of natural gas \& power," in 2014 47th Hawaii International Conference on System Sciences, pp. 2335-2343, IEEE, 2014.
[4] A. Rudkevich, A. Zlotnik, X. Li, P. Ruiz, A. Beylin, J. Goldis, R. Tabors, and R. Philbrick, "Evaluating benefits of rolling horizon model predictive control for intraday scheduling of a natural gas pipeline market," in Proceedings of the 52nd Hawaii International Conference on System Sciences, 2019.

[5] G. Freeman, J. Apt, and M. Dworkin, "The natural gas grid needs better monitoring," Issues in Science and Technology, vol. 34, no. 4, pp. 79-84, 2018.

[6] G. Freeman, Power plant-gas grid dependence. PhD thesis, Carnegie Mellon University, 2019.

[7] D. Hunger, "Analyzing gas and electric convergence mergers: A supply curve is worth a thousand words," Journal of Regulatory Economics, vol. 24, no. 2, pp. 161-173, 2003.

[8] O. Hart, J. Tirole, D. W. Carlton, and O. E. Williamson, "Vertical integration and market foreclosure," Brookings papers on economic activity. Microeconomics, vol. 1990, pp. 205-286, 1990.

[9] J. A. Ordover, G. Saloner, and S. C. Salop, "Equilibrium vertical foreclosure," The American Economic Review, pp. 127-142, 1990.

[10] P. L. Joskow and J. Tirole, "Transmission rights and market power on electric power networks," The Rand Journal of Economics, pp. 450-487, 2000.

[11] D. M. Newbery, "Power markets and market power," The energy journal, vol. 16, no. 3, 1995.

[12] J. B. Bushnell, E. T. Mansur, and C. Saravia, "Vertical arrangements, market structure, and competition: An analysis of restructured us electricity markets," American Economic Review, vol. 98, no. 1, pp. 237-66, 2008.

[13] C. Lo Prete and B. Hobbs, "A cooperative game theoretic analysis of incentives for microgrids in regulated electricity markets," Applied Energy, vol. 169, pp. 524-541, May 2016.

[14] M. Manea, “Cooperative games," 2017.

[15] S. Spiecker, "Modeling market power by natural gas producers and its impact on the power system," IEEE Transactions on Power Systems, vol. 28, no. 4, pp. 3737-3746, 2013.

[16] Z. Chen, Y. Zhang, T. Ji, Z. Cai, L. Li, and Z. Xu, "Coordinated optimal dispatch and market equilibrium of integrated electric power and natural gas networks with P2G embedded," Journal of Modern Power Systems and Clean Energy, vol. 6, pp. 495-508, may 2018.

[17] S. Fan, Z. Li, J. Wang, L. Piao, and Q. Ai, "Cooperative Economic Scheduling for Multiple Energy Hubs: A Bargaining Game Theoretic Perspective," IEEE Access, vol. 6, pp. 27777-27789, may 2018.

[18] "Natural gas consumption by end use." https: //www.eia.gov/dnav/ng/NG_CONS_SUM_ DCU_SNY_A.htm. 